Check for updates

Cite this: RSC Adv., 2017, 7, 21484

\section{Biosynthesis of zinc sulfide quantum dots using waste off-gas from a metal bioremediation process}

\author{
Angela J. Murray, $\uparrow^{\mathrm{a}}$ Jimmy Roussel, $\uparrow^{\mathrm{a}} \mathrm{J}$ John Rolley, ${ }^{a}$ Frankie Woodhall, ${ }^{a}$ \\ Iryna P. Mikheenko, ${ }^{a}$ D. Barrie Johnson, ${ }^{b}$ Jaime Gomez-Bolivar, ${ }^{c}$ \\ Mohamed L. Merroun ${ }^{c}$ and Lynne E. Macaskie (D)*a
}

\begin{abstract}
Dissimilatory reduction of sulfate, mediated by various species of sulfate-reducing bacteria (SRB) and a few characterized species of archaea, can be used to remediate acid mine drainage (AMD). Hydrogen sulfide $\left(\mathrm{H}_{2} \mathrm{~S} / \mathrm{HS}^{-}\right)$generated by SRB removes toxic metals from AMD as sulfide biominerals. For this, SRB are usually housed in separate reactor vessels to those where metal sulfides are generated; $\mathrm{H}_{2} \mathrm{~S}$ is delivered to AMD-containing vessels in solution or as a gas, allowing controlled separation of metal precipitation and facilitating enhanced process control. Industries such as optoelectronics use quantum dots (QDs) in various applications, e.g. as light emitting diodes and in solar photovoltaics. QDs are nanocrystals with semiconductor bands that allow them to absorb light and re-emit it at specific wavelength couples, shifting electrons to a higher energy and then emitting light during the relaxation phase. Traditional QD production is costly and/or complex. We report the use of waste $\mathrm{H}_{2} \mathrm{~S}$ gas from an AMD remediation process to synthesize zinc sulfide QDs which are indistinguishable from chemically prepared counterparts with respect to their physical and optical properties, and highlight the potential for a empirical process to convert a gaseous "waste" into a high value product.
\end{abstract}

Received 5th July 2016

Accepted 18th March 2017

DOI: 10.1039/c6ra17236a

rsc.li/rsc-advances to selectively precipitate transition metals as sulfides either within the bioreactor itself ${ }^{3}$ or within a separate reaction vessel. ${ }^{4}$ One advantage of mediating sulfate reduction in acidic liquors is that the end products are almost exclusively $\mathrm{H}_{2} \mathrm{~S}$ and $\mathrm{CO}_{2}$ rather than, at higher $\mathrm{pH}, \mathrm{H}_{2} \mathrm{~S} / \mathrm{HS}^{-}$and $\mathrm{HCO}_{3}{ }^{-} . \mathrm{H}_{2} \mathrm{~S}$ and $\mathrm{CO}_{2}$ are readily transferred in waste gas streams from bioreactors to other reactor vessels to facilitate off-line metal precipitation.

Contacting $\mathrm{H}_{2} \mathrm{~S}$ with many (chalcophilic) transition metal cations can, depending on factors such as $\mathrm{pH}$, generate metal sulfides. Nanoparticulate metal sulfides are a major current focus due to their potential applications in optoelectronic devices. ${ }^{5}$ Optoelectronics industries increasingly rely on quantum dots (QDs) for applications such as light emitting diodes and solar photovoltaics. QDs are nanocrystals with semiconductor bands that allow them to absorb light and reemit it intensely at specific wavelength couples. This property is confined to small nanoparticles (NPs) which allow electrons to be shifted to a higher energy and then emit light towards the red end of the spectrum during the relaxation phase. The QD elemental composition, and the presence of doping agent, determine its electronic band gaps and can be used to tune the QD to the desired red-shifted emission wavelengths. However traditional QD production at scale is costly and/or complex.

Zinc sulfide is a II-VI semiconducting material with a bandgap varying from $3.7 \mathrm{eV}$ (bulk material) to $4.2 \mathrm{eV}(\mathrm{NPs})^{6,7}$ with a large exciton energy ( $\sim 40 \mathrm{meV})$ which has been applied in devices such as flat panel displays and light emitting diodes. ${ }^{8}$ 
The QDs that comprise such materials consist of NPs usually synthesized and stabilized in such as way as to reduce agglomeration. This is important because the quantum yield (number of electrons released by a photocell per photon of incident radiation of a given energy) is higher by using NPs than that from larger particles, underpinning the former as the focus of current applications. ${ }^{8}$ Zinc is especially attractive in, for example, biological imaging due to its relatively low biological toxicity.

Quantum dot particles are usually synthesized chemically in organic solvent ${ }^{9}$ or in the presence of a surfactant or capping agent to prevent agglomeration. Typical organic and wet chemical methods of quantum dot production, e.g. aqueous colloidal synthesis, micro-emulsions etc. may have limited reproducibility and are costly (see ref. 10 for overview). Various options have been examined to reduce the high cost of synthesis of $\mathrm{ZnS}$ NPs at scale. Khani et al. ${ }^{11}$ used $\mathrm{ZnCl}_{2}$ and $\mathrm{Na}_{2} \mathrm{~S}$ with 2mercaptoethanol as the capping agent. Later, Senthilkumar et al. ${ }^{12}$ used $\mathrm{Zn}^{2+}$ solution with $\mathrm{Na}_{2} \mathrm{~S}$ and mercaptopropionic acid in the presence of tetrapropyl ammonium hydroxide with a refluxing step. The absorption edge was $315 \mathrm{~nm}$, the emission peak was $\sim 415 \mathrm{~nm}$ and the QD size was $\sim 4-5 \mathrm{~nm} .{ }^{12}$ A similar study produced $\mathrm{ZnS}$ QDs of size $3.5 \mathrm{~nm}$ using $\mathrm{Zn}^{2+}, \mathrm{Na}_{2} \mathrm{~S}$ and thiolactic acid, observing QD absorbance and emission at $279 \mathrm{~nm}$ and $435 \mathrm{~nm}$ respectively. ${ }^{13}$

In a preliminary test we established that $\mathrm{ZnS}$ made by gassing a complex bioleach liquor containing $\mathrm{Zn}^{2+}$ produced metal sulfide material with no optical property, probably due to the presence of a mixture of metals in the leachate and precluding a simple 'waste to QD product' process. This highlighted the need for a well-defined metal solution or segregation of metals from the waste, e.g. as described by Nancucheo \& Johnson. ${ }^{3}$ As an alternative to using waste metals, the objective of this study was to fabricate $\mathrm{ZnS}$ quantum dots by using a $\mathrm{Zn}^{2+}$ solution and by feeding excess $\mathrm{H}_{2} \mathrm{~S}$ from the off-gas from the sulfidogenic metal remediation process. We report the light emitting property of the resulting $\mathrm{ZnS}$ quantum dots in comparison with those made by published chemical methods requiring more complex synthetic procedures.

\section{Materials and methods}

\section{Microbial growth, $\mathrm{H}_{2} \mathrm{~S}$ production and formation of $\mathrm{ZnS}$}

For batch-tests, the sulfate-reducing deltaproteobacterium Desulfovibrio desulfuricans NCIMB 8307 was grown anaerobically in Postgate's medium C. Gas samples were withdrawn from the culture headspace, through a rubber septum, during (i) mid-late exponential growth phase, (ii) after $48 \mathrm{~h}$, and (iii) following culture ageing for several weeks with no further addition of nutrients. Test solutions $\left(40 \mathrm{~mL}\right.$ of $50 \mathrm{mM} \mathrm{ZnSO}_{4}$ in $50 \mathrm{mM}$ citrate buffer, pH 6.0) were put into $50 \mathrm{~mL}$ serum bottles, which were sealed with rubber septa, and the head space air was displaced with $\mathrm{N}_{2}$ gas bubbled through the mixture, allowing air to escape though a needle in the septum. Gas samples ( $5 \mathrm{~mL}$ per addition) from the $D$. desulfuricans culture were injected into zinc sulfatebuffer solution in the serum bottle under positive pressure, and bottles were shaken to facilitate mass transfer through the liquid phase. The pressure was released via insertion of a needle after every $10 \mathrm{~mL}$ of gas addition. The white precipitate obtained from several sequential injections was harvested by centrifugation, washed in water (purified by reverse osmosis) twice $(50 \mathrm{~mL})$ and the putative nanoparticles (NPs) were resuspended in aqueous dispersion. $\mathrm{ZnS}$ was also generated using a $\mathrm{N}_{2} / \mathrm{H}_{2} \mathrm{~S}$ gas stream from a low $\mathrm{pH}$ (4.0) continuous-flow sulfidogenic bioreactor, which used glycerol as the electron donor. ${ }^{4}$ Hydrogen sulfide generated by SRB in the reactor vessel was removed in a continuous stream of oxygen-free nitrogen. The gas flow rate was $185 \mathrm{~mL}$ $\min ^{-1}$, and the $\mathrm{H}_{2} \mathrm{~S}$ content was $0.1 \%$. This gas was fed into a bottle containing $40 \mathrm{~mL}$ of $50 \mathrm{mM}$ zinc sulfate/citrate solution (as above) for $5 \mathrm{~min}$ intervals, with shaking between dosings. Samples were stored in the dark $\left(4{ }^{\circ} \mathrm{C}\right)$ and examined as described for batch tests using $D$. desulfuricans. Experiments were carried out at least twice using separate batch preparations of $D$. desulfuricans or in two sampling periods from the continuous sulfidogenic reactor, separated by several weeks.

\section{Analysis of head gas of $D$. desulfuricans}

The head gas was analyzed by GC (Waters GCT Premier gas chromatograph) fitted with an orthogonal acceleration-flight of time mass spectrometer. The $\mathrm{m} / \mathrm{z}$ range was from 0 to 80 and $\mathrm{m} /$ $z$ values were compared with literature databases.

\section{Examination of material by use of scanning and transmission electron microscopy, X-ray powder diffraction (XRD) and analytical disc centrifuge}

For routine TEM examination, the samples were diluted in distilled water (1:5 vol/vol), $3 \mu \mathrm{L}$ of the diluted sample was applied onto copper grids (formvar coated $3 \mathrm{~mm}, 300 \mathrm{mesh}$ ) and left to air dry briefly. The grids were then carefully submerged in distilled water (to remove residual solute and prevent inorganic salt crystallization), and air dried. Samples were examined using a Jeol 1200-EX TEM (accelerating voltage $80 \mathrm{kV}$ ). Dried samples $\left(50{ }^{\circ} \mathrm{C}\right.$; desiccator $)$ were also washed with water $(2 \times 50$ $\mathrm{mL}$ ) and examined (vacuum mode) using an environmental scanning electron microscope (ESEM: Philips XL-30; $\mathrm{LaB}_{6}$ filament) fitted with a HKL EBSD system with NodrlysS camera, and an INCA EDS detector (Oxford Instruments) for energy dispersive X-ray analysis (EDS). High resolution transmission and scanning-transmission electron microscopy (TEM-STEM) were performed using a Tecnai F30 (FEI) instrument at a working voltage of $300 \mathrm{kV}$. TEM images and Selected Area Electron Diffraction (SAED) patterns were obtained with a coupled CCD camera (Gatan), whereas High Angle Annular Dark Field (STEM-HAADF) images were obtained with a HAADF detector (Fischione). In order to confirm the chemical composition of the materials, X-ray Energy Dispersive Spectra (EDS) were obtained with an EDAX detector. Lattice spacings were determined using "ImageJ" through profiling of high resolution HRTEM images and compared against lattice spacing of ZnS from the JCPDS 79-0043 database.

XRD analysis of powdered, dried samples was carried out using a Bruker D8 Advanced Powder X-ray Diffractometer; 2 theta range of $20-70^{\circ}$ with step of $0.02^{\circ} ; 25^{\circ} \mathrm{C}$. 
Particle size distribution analysis was performed on aqueous NP suspensions using differential centrifugation (CPS 24000 analytical disc centrifuge: Analytik Ltd). Samples were analyzed via injection into the centrifuge with a sucrose gradient (8-24\%) applied to separate the particles by size on a disc rotating at $22000 \mathrm{rpm}$. Detection of the number of particles was estimated using a light detector on the edge of the disc and the particle size was estimated using software supplied by the manufacturer. The lowest limit of detection was $\sim 5 \mathrm{~nm}$.

\section{Spectroscopic analysis of materials}

Prior to analysis, samples were diluted to an absorbance of 0.20.8. The absorption, excitation and emission wavelengths were determined using, respectively, a Cary 50 Eclipse UV-visible spectrometer (scanning range from $200 \mathrm{~nm}$ to $800 \mathrm{~nm}$ ) using $3 \mathrm{~mL}$ quartz cuvettes and a FLSP920 Time Resolved Spectrometer in a quartz cuvette over a range of excitation wavelengths from $250 \mathrm{~nm}$ to $450 \mathrm{~nm}$; emission peaks were quantified from $350 \mathrm{~nm}$ to $650 \mathrm{~nm}$ for the emission wavelength by use of a $340 \mathrm{~nm}$ filter.

\section{Reproducibility of data}

Experiments were carried out multiple times in succession using separate bacterial preparations by way of two research teams separated by $\sim$ two years, with similar results.

\section{Results and discussion}

\section{Effect of culture age and headspace gas analysis}

Identical results were obtained using head gas samples withdrawn from actively growing cultures of $D$. desulfuricans during mid-late exponential growth, after $48 \mathrm{~h}$ and by using aged cultures after several weeks. Analysis of the culture head gas (Fig. 1) shows peaks at $m / z$ values of 33.0-36.0 which correspond to the fragmentation pattern of $\mathrm{H}_{2} \mathrm{~S}$. No dimethyl sulfide $\left(\mathrm{CH}_{3} \mathrm{CH}_{3} \mathrm{~S} ; m / z=62\right)$ or dimethyl disulfide $\left(\mathrm{CH}_{3} \mathrm{CH}_{3} \mathrm{~S}_{2} \mathrm{~m} / z=94-\right.$ 97), which could have provided a 'capping' function, were detected. An unidentified peak at $m / z=40.0$ was attributed to background noise from the instrument and additional peaks at $\mathrm{m} / \mathrm{z}$ of 63.9 and $44-45 \mathrm{~m} / \mathrm{z}$ were not identified further. The concentration of $\mathrm{H}_{2} \mathrm{~S}$ in the headspace of the batch cultures was not quantified.

\section{Examination of the material made via culture head gas}

A turbid white suspension was formed immediately after addition of $D$. desulfuricans off-gas into the serum bottle (Fig. 2a) which settled only slowly (several days) under gravity. Similar results were obtained using the batch cultures and the sulfidogenic culture off-gas. A colloidal suspension was similarly observed by Bol et al. (2002) ${ }^{\mathbf{1 4}}$ during chemical synthesis of ZnS nanoparticles. The expected compounds formed under anoxic conditions are amorphous $\mathrm{ZnS}$, crystallized sphalerite (a gemstone) or wurtzite, a zinc iron sulfide mineral. However, the former is yellow, orange or green while the latter is orange/ reddish brown to black, whereas the material formed here was white (Fig. 2a). The minerals have crystallite diameters on the micrometer scale. ${ }^{15}$ During preparation of the material for solid state analysis (by XRD; below) a large agglomerated structure was apparent (by ESEM) after centrifugation (Fig. 2b). Dried precipitate, made from the suspension and viewed by ESEM (Fig. 2c, inset) and TEM at $80 \mathrm{kV}$ (Fig. 2d, inset) appears to comprise nanoparticles of size estimated at $20-40 \mathrm{~nm}$, with smaller NPs visible within the larger ones (Fig. 2c inset). A size distribution analysis of the small NP inclusions was precluded

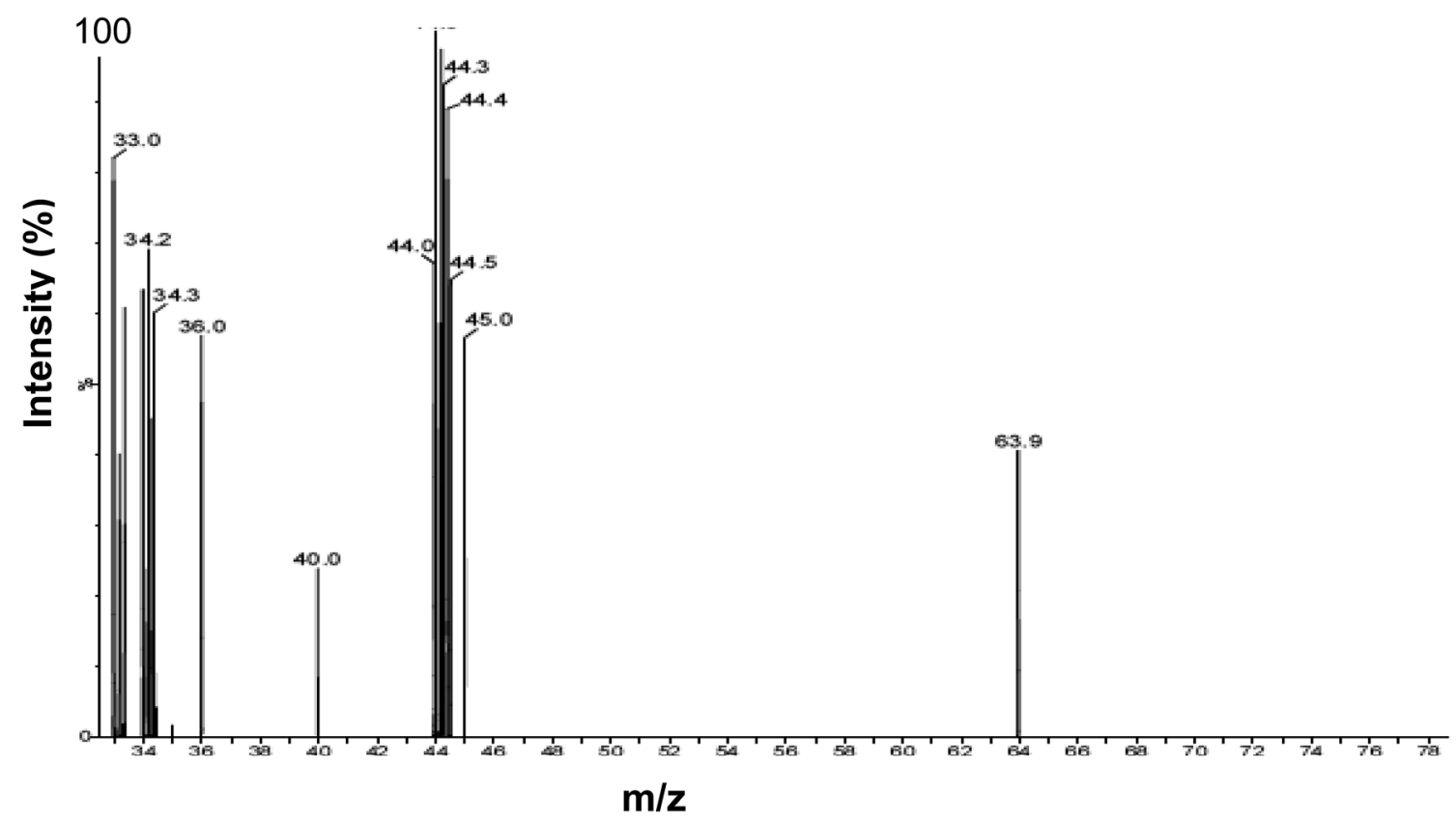

Fig. 1 Analysis of $D$. desulfuricans culture headspace gas using GC-MS. Peaks $(\mathrm{m} / \mathrm{z})$ were assigned by reference to MS databases. 
due to their lack of definition (Fig. 2c, inset) while the larger NPs were not sufficiently well separated for analysis with confidence (Fig. 2d, inset).

The putative zinc sulfide nano-material (Fig. 2a and b) was formed identically in contact with both sources of biological head gas which contained $\mathrm{H}_{2} \mathrm{~S}$. Energy dispersive X-ray analysis confirmed the presence of both zinc and sulfur (Fig. 2c); the mass ratio was determined (mass percentage) as $\mathrm{Zn}: \mathrm{S}$ of $2: 1$.
Since the atomic weight of zinc $\left(65.4 \mathrm{~g} \mathrm{~mol}^{-1}\right)$ is about twice that of sulfur $\left(32.1 \mathrm{~g} \mathrm{~mol}^{-1}\right)$ the ratio of $\mathrm{Zn}: \mathrm{S}$ was $1: 1$, supporting the identification of the material as ZnS. To confirm this, XRD analysis (Fig. 2d) showed a crystalline structure with three distinct peaks at 2 theta of $28.9^{\circ}, 47.6^{\circ}$ and $57.5^{\circ}$, matching with the $\mathrm{ZnS}$ reference and confirming the identity of the material. $\mathrm{XRD}$ also gives information about the crystal structure. Several studies ${ }^{11,12,16,17}$ have shown that the three peaks can be assigned
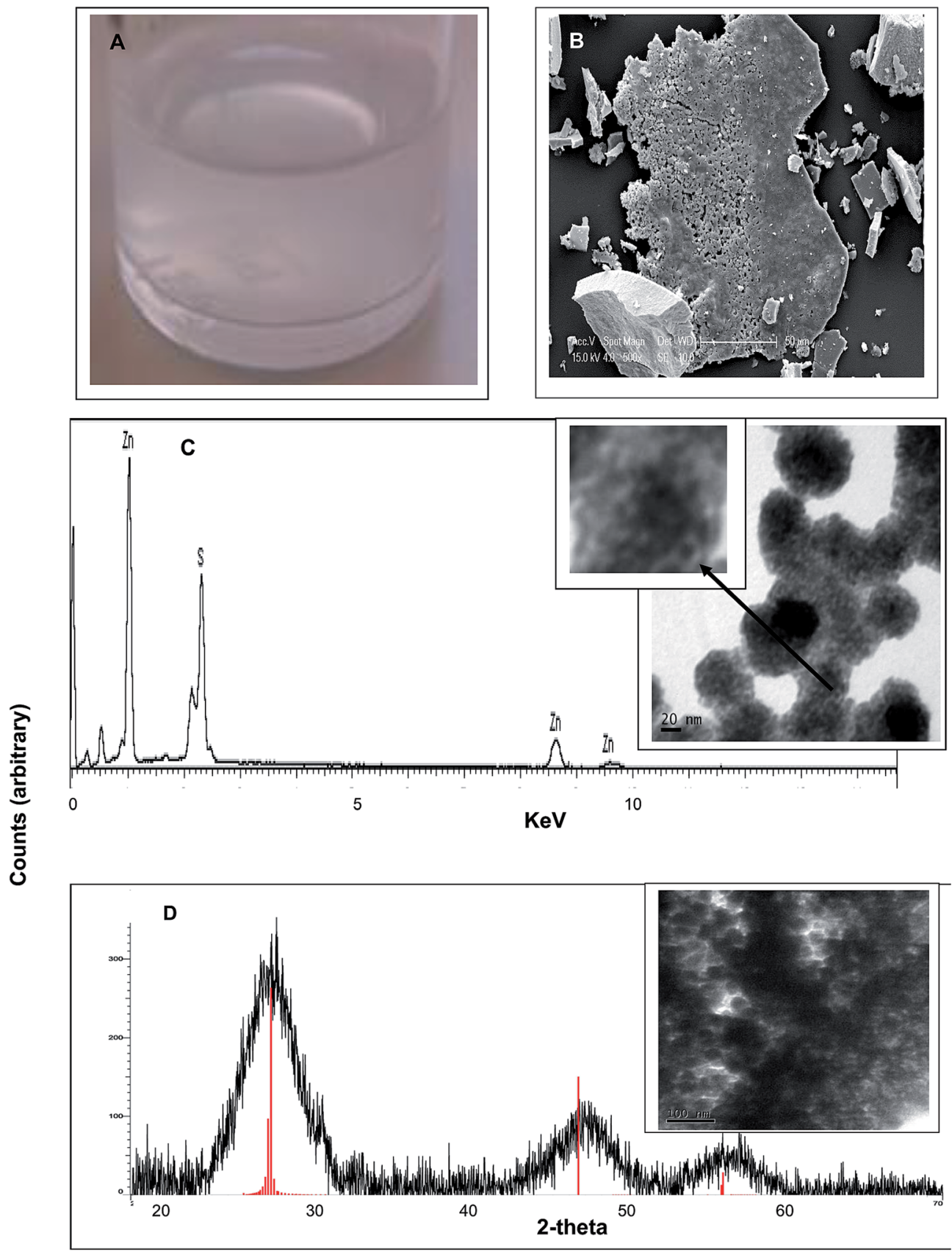

Fig. 2 Material formed after addition of $D$. desulfuricans head-gas to a mixture of $50 \mathrm{mM} \mathrm{ZnSO}_{4}$ and $50 \mathrm{mM}$ citrate buffer, pH 6 . Similar results were obtained using the sulfidogenic bioreactor off-gas. (A) White precipitate formed in the solution. (B) Appearance of the material by ESEM following centrifugation to make a pellet. (C) EDS analysis (under ESEM) of specimen microareas with peaks assigned to Zn and S. Inset: appearance of the material by TEM under accelerating voltage of $80 \mathrm{kV}$ (for good contrast ${ }^{25}$ ) note the speckled appearance of individual nanoparticles (bar is $20 \mathrm{~nm}$ ). (D) X-ray powder diffraction analysis of the bulk material shown inset (bar is $100 \mathrm{~nm}$ ). Vertical lines are reference peaks for ZnS (file: 04-017-5723). 
to the reflection of a cubic crystal structure of $\mathrm{ZnS}$ with $c(111)$ at $28.9^{\circ}, c(220)$ at $47.6^{\circ}$ and $c(311)$ at $57.5^{\circ}$. The XRD powder pattern was also used to calculate the crystalline domain size by application of the Debye-Scherrer equation. ${ }^{18}$ The average size from the three peaks gave a diameter of $2.3 \mathrm{~nm}$. This value was similar to that $(1.5-3.0 \mathrm{~nm})$ found during XRD analysis of several ZnS materials ${ }^{\mathbf{1 1 , 1 6}}$ and it was concluded that ZnS nanoparticles were formed. The NP size found by XRD $(2.3 \mathrm{~nm})$ was smaller than the NPs visible in Fig. 2c (inset) and was in accordance with the small NPs within the agglomerations (Fig. 2c, inset, arrowed). The size and appearance of the NPs by electron microscopy is in accordance with those reported by Ramachandran et al. ${ }^{19}$ obtained at an accelerating voltage of 30$50 \mathrm{kV}$; these authors estimated the small NP size of $\sim 6 \mathrm{~nm}$ with aggregation attributed to van der Waals forces between the small particles to form the larger bodies ${ }^{\mathbf{1 9}}$ while similar structures were observed by Shahid et al. ${ }^{20}$ and also by Shin et al. ${ }^{21}$ in the case of CdSe/ZnS core-shell QDs (ZnS shell).

\section{Characterization of nanoparticles using differential centrifugal analysis}

XRD is a 'bulk' method that gives information about average nanoparticle sizes but not size distributions. Size distribution was not possible to analyze by direct examination (data from several authors: above). Moreover, the samples were dried for EM examination. Particle size distribution analysis used differential centrifugal sedimentation and wet samples, confirming the presence of NPs in the suspensions (Fig. 3), of size distribution from below $10 \mathrm{~nm}$ to $20 \mathrm{~nm}$. The mean NP size of those detected (by number count, ignoring those below $5 \mathrm{~nm}$ ) was $13 \mathrm{~nm}$; the lowest detection limit of the analytical disc centrifuge under the conditions used is $\sim 5 \mathrm{~nm}$ so an accurate analysis of small NPs was not possible. The average size, of those that could be determined by this method (Fig. 3), was within the range (5-20 $\mathrm{nm}$ ) of $\mathrm{ZnS}$ QDs produced in earlier studies $^{6,22}$ as well as the measurement by XRD ( $\sim 2-3 \mathrm{~nm}$ : above) aligning with other reports (3.5 nm (ref. 12 and 13)). The actual size deduced is governed to some extent by the method used but this method of size distribution analysis suggests the absence of

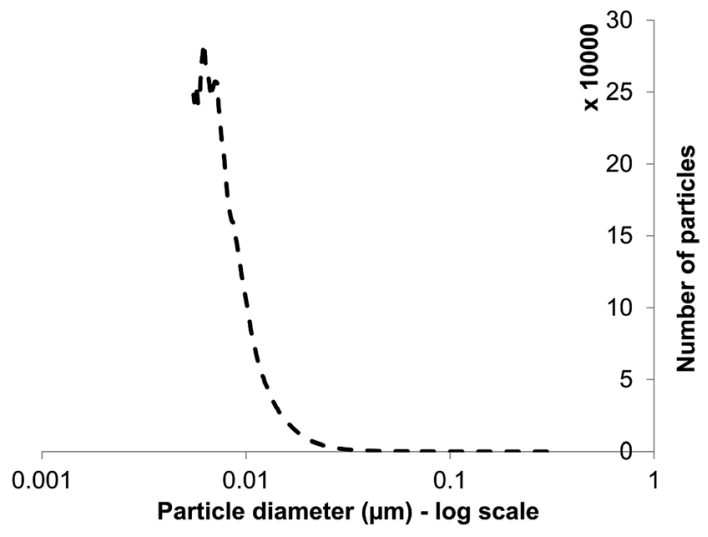

Fig. 3 Particle size distribution (number of particles at each size) for ZnS quantum dots (lower detection limit: $5 \mathrm{~nm}$ ) obtained by differential centrifugal sedimentation analysis. large NPs or agglomerations, contrasting with Fig. 2c and $\mathrm{d}$ (insets). Since the analytical disc centrifuge was used at near to its detection limit these results may be subject to error in the case of small NPs but confirm both direct observation of the small NPs within agglomerations (Fig. 2c, inset), and those from XRD analysis (Fig. 2d); most of the particles were suggested to be between 3-15 nm diameter. The strength of the centrifugal analysis is that the suspension can be examined dynamically, i.e. in the native state without drying or other preparation, which would also enable the evolution of NPs to be followed in real time or to quantify the effects of capping agents in a way that electron microscopy cannot. The 'snapshot' of Fig. 3, at the specified sucrose gradient and rotating disc speed, revealed only $\sim 5 \%$ of the NPs to be of sizes more than $20 \mathrm{~nm}$ and approximately $50 \%$ of them of $\sim 10 \mathrm{~nm}$, in apparent contradiction to the TEM data. It is not possible to obtain a size distribution of the smallest NPs; the rate of NP sedimentation and analysis time is controlled by various factors both sample based (size and density) and instrument based (disc speed, sedimentation volume/depth and fluid viscosity). It is possible to size particles down to 3-4 nm using particles of high density e.g. gold nano-particles (density $19.3 \mathrm{~g} \mathrm{~cm}^{-3}$ ) but not attainable with $\mathrm{ZnS}$ (density $<10 \mathrm{~g} \mathrm{~cm}^{-3}$ ). Even with a long sedimentation time data become unreliable due to Brownian motion effects between sedimenting particles and fluid/solvent ( $\mathrm{H}$. Vegad, personal communication).

We suggest that the centrifugal forces in the sucrose density gradient could be sufficient to overcome the van der Waals forces holding the agglomerations. However, while van der Waals forces are simple to calculate between parallel surfaces of $>10 \mathrm{~nm}$ apart, a calculation between only two nanospheres $<5 \mathrm{~nm}$ apart becomes difficult, ${ }^{23}$ and, with the agglomerations comprising many small NPs, and additional consideration of the differential centrifugal force, the calculation is well beyond the scope of this study. An alternative explanation is that while drying (used in the EM method but not in the differential centrifugal method) the small NPs are pulled together by water surface tension force which causes them to agglomerate artifactually; hence the centrifugal method gives a more accurate representation. In support of this, the technique is used widely to characterize latexes and emulsions, agglomerates and aggregates such as protein clusters, dimers, trimers and tetramers of proteins and virus particles. All of these are held together by van der Waals forces yet the observed peaks for these materials correspond to the different sizes (e.g. ref. 24) i.e. the centrifugal force does not have a disrupting effect on these entities and there is no reason to suggest that ZnS NPs should be any different.

We conclude that the use of electron microscopy to obtain particle size distributions has limitations in the case of $\mathrm{ZnS}$; even with a high contrast image (e.g. ref. 19) the small NPs are insufficiently well defined and well separated to facilitate reliable computational image analysis. The differential centrifugal sedimentation method can give a size distribution profile with a cutoff of about 5-6 $\mathrm{nm}$ and hence neither method is satisfactory. An average $\mathrm{ZnS}$ NP size $(2.4 \mathrm{~nm})$ was back-calculated from the absorption peak using Brus' effective model; ${ }^{20}$ this 
analysis method agrees with the XRD data we report (above) but, again, the calculation from optical data does not inform about the NP size distribution.

\section{Characterization by high resolution TEM}

In order to obtain high resolution data via HRTEM an accelerating voltage of $300 \mathrm{kV}$ was used. The EDS data obtained thus (not shown) confirmed that shown in Fig. 2c. Egerton ${ }^{25}$ noted that while high voltages give high resolution for structural analysis lower voltages can be useful to provide more contrast for size and morphology analysis (as above). Accordingly, under HRTEM the details of the NPs were lost (Fig. 4a and b) but fine structure is apparent in Fig. 4c which shows the lattice fingers in crystalline material with lattice interplanar spacings of $0.32 \mathrm{~nm}$ corresponding to the $\mathrm{ZnS}$ (111) facet and $0.196 \mathrm{~nm}$ corresponding to the ZnS (220) facet. Examination by SAED (Fig. 4d) shows diffraction rings indexed to the (111), (220) and (311) planes to the cubic phase; both sets of data are in agreement with the XRD data in Fig. 3d and are consistent with other reports. ${ }^{19-21,26}$

\section{Optical properties of biogenic zinc sulfide nanoparticles and potential for use as quantum dots}

The presence of nano-material per se is not sufficient to obtain quantum dots; the compound in suspension must possess a semi-conductor band allowing the absorption of light energy by electron displacement. During the electron relaxation, the energy stored is released as light emission at a specific wavelength; the characteristic of the band is determined by its specific absorption and emission wavelengths.

The zinc sulfate/citrate solution was sparged with the $\mathrm{H}_{2} \mathrm{~S} / \mathrm{N}_{2}$ gas from the sulfidogenic bioreactor for increasing times up to
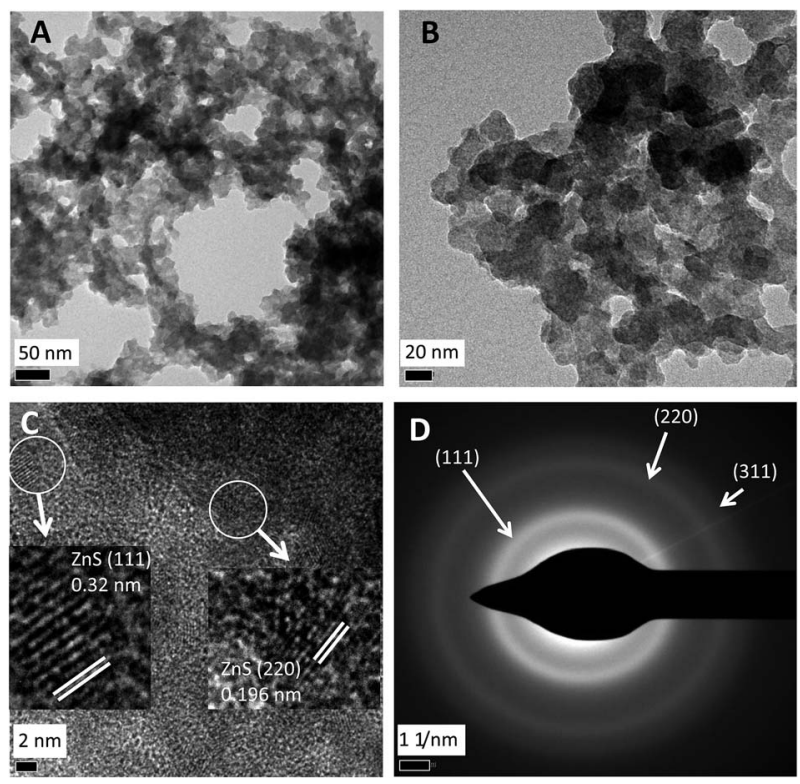

Fig. 4 High resolution TEM study of ZnS quantum dots (FEI Tecnai F30; $300 \mathrm{kV}$ ). (A and B) High-resolution TEM images of ZnS nanoparticles. (C) HRTEM of ZnS crystals with inserts revealing lattice spacing. (D) Selected area electron diffraction patterns of ZnS nanoparticles.
$35 \mathrm{~min}$. The emission peak intensity of the $\mathrm{ZnS}$ material obtained (see below) was proportional to the period of gassparging. Saturation, after $\sim 25 \mathrm{~min}$, had no apparent effect on the emission peak position of the $\mathrm{ZnS}$ product. The exposure time did not therefore influence the QD size, and particles did not start to aggregate, and thereby lose their optical properties.

Examination of both sets of materials by UV-visible spectroscopy showed absorption in a band width of $270-320 \mathrm{~nm}$ (Fig. 5) with a peak at $290 \mathrm{~nm}$, suggesting that the suspended NPs have an electronic gap band of $4.2 \mathrm{eV}(E=h c / \lambda ; E$ is photon energy $h$ is Planck constant, $\lambda$ wavelength and $c$ speed of light). A ZnS nanoparticle has a high band gap and so absorbs in the UV part of the spectrum while macro-ZnS (lower band gap) absorbs in the visible spectrum, via deduction from the energy band gap difference between macro and nano size $(E=3.6 \mathrm{eV}$ for macrostructure and $4.2 \mathrm{eV}$ for a nanostructure).

This absorption wavelength (Fig. 5) was used to excite the electron present on the valence orbital onto the first excited orbital. The light-emission wavelength, resulting from the release of the electron to the valence band, was determined, with an emission peak of $410 \mathrm{~nm}$. The maximum was conserved at an excitation wavelength of 290-305 nm (optimally at 290 nm) (Fig. 5 and 6).

Light emission from the suspended ZnS NPs was observed in the visible spectrum (excitation wavelength 290-310 nm) with photoluminescence in a broad spectrum from $350 \mathrm{~nm}$ to $500 \mathrm{~nm}$, around the peak emission at $410 \mathrm{~nm}$. Several different excitation wavelengths (270-320 nm) were used to confirm that the emission was from this band gap and to determine the best excitation wavelength $(290 \mathrm{~nm})$ and the couple excitation/emission wavelengths of 290/410 $\mathrm{nm}$ for the suspended $\mathrm{ZnS}$ nanoparticles.

The results reported here are in accordance with other authors; Shahid et al. ${ }^{20}$ noted an emission peak for cubic phase ZnS QDs at $439 \mathrm{~nm}$ (excitation at $260 \mathrm{~nm}$ ), discussing their results in terms of strong quantum confinement effects, the quantum confinement arising from the small size of the ZnS QDs comparable to the excitonic Bohr diameter; ${ }^{20}$ with cubic phase $\mathrm{ZnS}$ this is $\sim 2.4 \mathrm{~nm} .{ }^{27}$ Further, Shahid et al. ${ }^{20}$ calculated the size of the QDs to be $2.4 \mathrm{~nm}$ from the absorption peak using Brus' effective model which is in agreement with the results obtained here using XRD. However the optical data was not

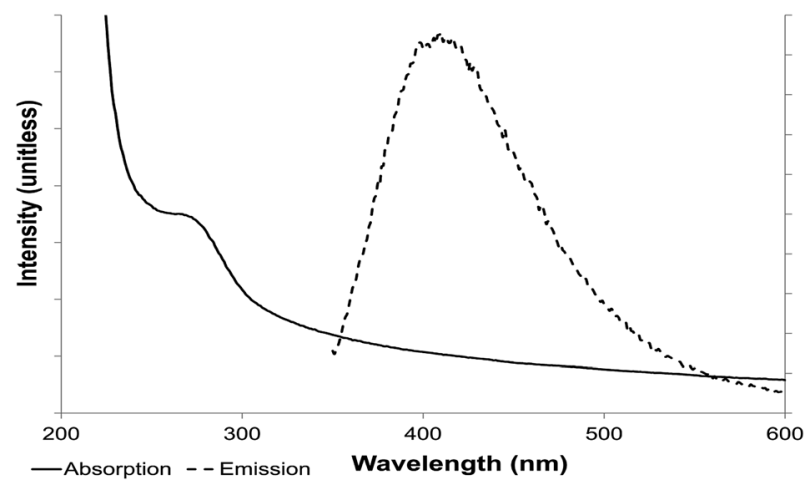

Fig. 5 Photoluminescence properties of ZnS quantum dots. Absorption (solid line) and emission (dotted line) spectroscopy. 


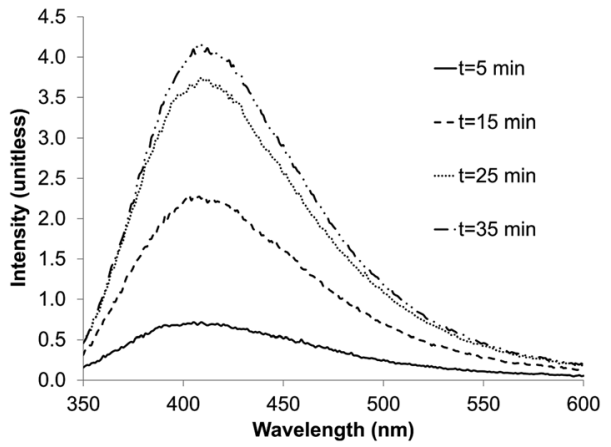

Fig. 6 Effect of exposure time to AMD sulfidogenic bioreactor off-gas on the emission peaks for biogenic ZnS quantum dots.

used to inform about NP size distribution. As shown in Fig. 5, the emission peak $^{\mathbf{2 0}}$ was broad with no strongly defined maximum. Ramachandran et al. ${ }^{19}$ noted an emission peak at $424 \mathrm{~nm}$ (excitation at $320 \mathrm{~nm}$ ) for a $\mathrm{ZnS} /$ graphene composite, i.e. a slight red-shift in the excitation and emission wavelengths as compared to the work reported here; they suggested that the electronic properties of the $\mathrm{ZnS}$ are modified by the presence of electrically conductive graphene, also noting that the photoluminescence intensity of the composite was higher than that of pure ZnS NPs, attributed to an energy transfer from graphene to ZnS.

In two cases ${ }^{20,26}$ the emission peak for ZnS QDs was similarly broad to that shown in Fig. 5; Ramachandran et al. ${ }^{19}$ reported a narrower peak (412-437 $\mathrm{nm}$ ) but this was not attributable to the presence of graphene since the peaks obtained by ZnS with and without graphene were identical. These works, in accordance with the current study, have focused on the light emitting properties of ZnS QDs but have tended to overlook the quantum yield which is also important with respect to future applications. The QY of the biogenic material is under current consideration.

\section{Conclusions}

It is concluded that, by a very simple one-step method, $\mathrm{ZnS}$ quantum dots analogous to those produced commercially and as reported by numerous authors, can be made in a scalable way via using waste biogenic $\mathrm{H}_{2} \mathrm{~S}$. This produces both a safe disposal route for this toxic gaseous byproduct of AMD remediation and a potentially valuable material. The $\mathrm{Zn}^{2+}$ is not placed in contact with the bacteria and hence metal toxicity to them is not an issue, while the product contains no bacterial cells. Harnessing a continuous bioprocess for $\mathrm{H}_{2} \mathrm{~S}$ production would overcome potential issues of economic QD production at scale and this approach would be applicable also to making sulfide quantum dots of other group IIB metals.

\section{Acknowledgements}

This work was supported by NERC (Grant No. NE/L014076/1 and NE/L014114/1). We acknowledge with thanks useful discussions with Prof Z. Pikramenou (University of
Birmingham) and H. Vegad (Analytik). This research was supported through Birmingham Science City (West Midlands Centre for Advanced Materials Project 2), with support from Advantage West Midlands and part funded by the European Regional Development Fund.

\section{References}

1 P. Saari and M. Riekkola, J. South. Afr. Inst. Min. Metall., 2012, 112, 1013-1020.

2 I. Nancucheo, S. Hedrich and D. B. Johnson, Mineral. Mag., 2012, 76, 2683-2692.

3 I. Nancucheo and D. B. Johnson, Microb. Biotechnol., 2012, 5, 34-44.

4 S. Hedrich and D. B. Johnson, Environ. Sci. Technol., 2014, 48, 12206-12212.

5 P. F. Smet, I. Moreels, Z. Hens and D. Poelman, Materials, 2010, 3, 2834-2883.

6 J. Huang, Y. Yang, S. Xue, B. Yang, S. Liu and J. Shen, Appl. Phys. Lett., 1997, 70, 2335-2337.

7 A. A. Khosravi, M. Kundu, L. Jatwa, K. Deshponde, U. A. Bhagwart, M. Sasatry and S. K. Kulkami, Appl. Phys. Lett., 1995, 67, 2702-2704.

8 C. H. Lai, M. Y. Lu and L. J. Chen, J. Mater. Chem., 2012, 22, 19-30.

9 R. L. Johnston and J. P. Wilcoxon, Metal Nanoparticles and Nanoalloys, Elvesier, London, 2012, vol. 3.

10 N. Dixit and H. P. Soni, Superlattices Microstruct., 2014, 65, 344-352.

11 O. Khani, H. R. Rajabi, M. H. Yousefi, A. A. Khosravi, M. Jannesari and M. Shamsipur, Spectrochim. Acta, Part A, 2011, 79, 361-369.

12 K. Senthilkumar, K. Ramamurthi, T. Kalaivani and V. Balasubramanian, Indian J. Adv. Chem. Sci., 2013, 2, 1-5.

13 A. Mandal, A. Dandapat and G. De, Analyst, 2012, 137, 765772.

14 A. A. Bol, J. Ferwerda, J. A. Bergwerff and A. Meijerink, J. Lumin., 2002, 99, 325-334.

15 E. Pelletier, P. Ilipila and D. Fowle, Appl. Geochem., 2011, 26, 1673-1680.

16 D. J. Jovanovic, I. L. Validzic, I. A. Jankovic, N. Bibic and J. M. Nedeljkovic, Mater. Lett., 2007, 61, 4396-4399.

17 N. K. Abbas, K. T. Al-Rasoul and Z. J. Shanan, Int. J. Electrochem. Sci., 2013, 8, 3049-3056.

18 A. L. Patterson, Phys. Rev. B: Condens. Matter Mater. Phys., 1939, 56, 978-982.

19 R. Ramachandran, M. Suranya, P. Kollu, B. P. C. Ragupathy, S. K. Jeong and A. N. Grace, Electrochim. Acta, 2015, 178, 647657.

20 R. Shahid, M. S. Toprak, H. M. A. Soliman and M. Muhammed, Cent. Eur. J. Chem., 2012, 10, 54-58.

21 H. Shin, D. Jang, J. Hwang, Y. Jang, M. Cho and K. Park, J. Mater. Sci.: Mater. Electron., 2014, 25, 2047-2052.

22 S. Lee, D. Sond, J. Lee, S. Kim, I. Y. Park and M. S. Won, Mater. Sci. Eng., B, 2003, 103, 241-245.

23 Y. Luo, R. Zhao and J. B. Pendry, Proc. Natl. Acad. Sci. U. S. A., 2014, 111, 18422-18427. 
24 H. Amiri, L. Bordonali, A. Lascialfari, S. Wan, M. P. Monopoli, I. Lynch, S. Laurentg and M. Mahmoudi, Nanoscale, 2013, 5, 8656-8665.

25 R. F. Egerton, Ultramicroscopy, 2014, 145, 85-93.
26 X. Huang, M. G. Willinger, H. Fan, Z. I. Xie, A. K. Hoffmann, F. Girgsdies, C. S. Lee and X. M. Meng, Nanoscale, 2014, 6, 8787-8795.

27 H. Tang, G. Xu, L. Weng, L. Pan and L. Wang, Acta Mater., 2004, 52, 1489-1494. 